\title{
Capital Social y Políticas de Atención Primaria de la Salud.
}

\section{Capital Social e Políticas de Atenção Primária à Saúde. Social Capital and Primary Health Care Policies.}

Ernesto Pablo BÁSCOLO ${ }^{1}$

Natalia YAVICH ${ }^{2}$

RESUMEN: El objetivo de este trabajo es analizar la influencia de la configuración del capital social de las políticas de atención primaria de la salud (APS) sobre las condiciones de acceso a los servicios de salud. Se llevó a cabo un estudio de casos múltiples (Cochabamba-Bolivia, Rosario y Vicente López-Argentina) retrospectivo (1990-2008). El concepto de capital social fue entendido como el resultado de la relación entre Estado y sociedad. En Rosario se lograron mejoras sustantivas en las condiciones de acceso a partir de la creación de nuevas estructuras en donde profesionales de APS establecieron vínculos de cooperación con actores del Estado municipal, movimientos sociales y gestores y médicos especialistas de hospitales. En Vicente López, las mejoras en las condiciones de acceso a los servicios de salud fueron más limitadas debido a que los cambios en la relación Estado y sociedad solo involucraron a profesionales del primer nivel de atención sin mecanismos de cooperación estables con actores claves de los hospitales, el nivel municipal y los movimientos sociales. Por último, en Cochabamba, las mejoras en las condiciones de acceso fueron marginales debido a que los profesionales del sistema de servicios de salud se opusieron a la alianza establecida entre los movimientos sociales y los actores del Estado municipal para generar cambios en el sistema de salud. Este estudio permite reconocer la influencia de la configuración entre Estado y sociedad para modelar las relaciones de poder que subyacen al proceso de implementación de las reformas del sector salud y sus resultados.

Palabras clave: Atención Primaria de Salud, Accesibilidad a los Servicios de Salud, Capital Social, Política Organizacional, Organización Institucional.

ABSTRACT: The aim of this paper it to analyze the influence of the configuration of the primary health care (PHC) policies social capital on the healthcare access conditions. We conducted a retrospective (1990-2008) multiple case study (Rosario-Argentina, Vicente López-Argentina

1 CONICET / Universidad Nacional de Rosario-Centro de Estudios Interdisciplinarios. Argentina. E-mail: ebascolo@gmail.com

2 CONICET / Universidad Nacional de Rosario-Centro de Estudios Interdisciplinarios, Argentina. E-mail: nyavich@ gmail.com 
and Cochabamba, Bolivia). We understand Based on the compression of social capital as a result of the relationship between the State and Society, the results shows that Rosario's PHC policies promoted substancial improvements in healthcare access through the emergence of new structures in which APS professionals established cooperative relationships with municipal state actors, social movements and hospitals' managers and physicians. Vicente López, improvements in healthcare access conditions were more limited because changes in the relationship between state and society only involved primary care level professionals without stable mechanisms of cooperation with key actors of hospitals, municipal govermental structures and social movements. Finally, in Cochabamba, improvements in healthcare access conditions were marginal because the health services professionals opposed the alliance established between social movements and local state actors to generate changes in the health system. This study recognize the influence of the configuration of the State and the society to shape the power relations that underlie the process of implementing health sector reforms and their results.

Key words: Primary Health Care, Health Services Accessibility, Social Capital, Organizational Policy, Institutional Organization.

RESUMO: O objetivo deste artigo é analisar a influência da definição de políticas de capital de atenção primária à saúde (APS) sobre as condições de acesso aos serviços de saúde. Para este efeito, é realizado um estudo retrospectivo de casos múltiplos (Rosario-Argentina, Vicente LópezArgentina e Cochabamba, Bolívia) (1990-2008). O conceito de capital social foi entendido como o resultado da relação entre Estado e sociedade. Em Rosario melhorias substanciais nas condições de acesso foram obtidos a partir do criação de novas estruturas nas quais os profissionais APS estabeleceram laços de cooperação com representantes locais do Estado, movimentos sociais e gestores de hospitais e médicos especialistas. Em Vicente Lopez, as melhorias nas condições de acesso aos serviços de saúde foram mais limitados porque as mudanças na relação entre Estado e sociedade envolveram apenas os profissionais de nível dos cuidados primários sem mecanismos de cooperação estável com atores-chave hospitais, municipais e movimentos sociais. Finalmente, em Cochabamba, a melhoria das condições de acesso foram marginal, porque os profissionais do sistema de saúde se opôs à aliança estabelecida entre os movimentos sociais e representantes locais do Estado para gerar mudanças no sistema de saúde. Este estudo permite reconhecer a influência da configuração do Estado e da sociedade para moldar as relações de poder que permeiam o processo de implementação de reformas no sector da saúde e seus resultados.

Palavras-chave: Atenção Primária à Saúde, Acesso aos Serviços de Saúde, Capital Social, Política Organizacional, Organização Institucional.

\section{INTRODUCCIÓN}

En las dos últimas décadas el concepto de capital social ha sido una dimensión conceptual que ha adquirido relevancia para discutir la calidad de las instituciones y las condiciones de salud de la población. Mucha producción académica ha crecido dando soporte a la relación positiva del capital social sobre las condiciones de sociales de la población ${ }^{1-4}$. Al mismo tiempo, se ha 
generado un interesante debate teórico y metodológico sobre la aplicación del concepto de capital social en sectores y políticas sociales, entre los cuales la salud ha sido un campo de controversia especial ${ }^{5-8}$.

Este análisis procura ir más allá de reconocer la influencia de capital social sobre las condiciones de salud de la población. Un renovado interés se ubica en reconocer la relación del capital social como categoría que aborda la organización social de la comunidad y como un factor asociado a los procesos políticos e institucionales de políticas sociales en el sector salud y que contribuye a las condiciones de acceso a los servicios de la población.

La producción académica que argumenta a favor del papel del capital social sobre las condiciones de acceso de los servicios de salud tiende a concentrar el análisis en esta relación en términos causales, considerando a la primera (el capital social) como atributo institucional y variable independiente, condicionando los resultados del acceso como variable dependiente ${ }^{9-10}$.

Una de las críticas más importantes de este argumento, radica que esta relación no se produce en el vacío, sino más bien en el contexto de procesos políticos que integra la relación del Estado y la Sociedad Civil. Esta visión crítica sostiene que el capital social carece de poder explicativo para analizar cómo se construyen y sostienen las condiciones que afectan la problemática social y las intervenciones para superarla ${ }^{9-10}$. La caracterización y el rol del capital social podrían ser analizados en función del proceso social y político que sostiene el proceso de recomposición institucional, en donde es necesario reconocer las relaciones entre actores que hacen a la relación entre Estado y sociedad civil como ingrediente central tanto del proceso de implementación de las políticas públicas, como de la recomposición del campo institucional que se pretenden transformar.

En esta línea, el capital social debería ser considerada como una dimensión incluida en el análisis del proceso político e institucional de iniciativas de APS, y por lo tanto asumiendo los cambios de la relación Estado sociedad como procesos subyacentes en las políticas de públicas de extensión de la protección social en salud ${ }^{10}$.

Los interrogantes centrales de este trabajo pueden expresarse de la siguiente forma. ¿De qué manera el concepto de capital social puede aplicarse al análisis de las políticas de salud? Más específicamente: ¿cómo el concepto de capital social puede contribuir para entender los procesos de recomposición institucional de los sistemas de salud en un contexto de expansión de políticas de protección social en salud?

Para abordar este tema, se analizan casi dos décadas de iniciativas de políticas de APS en tres municipios, con diferentes dinámicas de la relación Estado y sociedad, y con diferentes alcances en las mejoras en el acceso a los servicios de salud.

La estructura de este artículo es la siguiente. En primer lugar, se presenta el concepto de capital social y su aplicación en el sector salud. Esta sección comprende los argumentos que sustentan el 
debate sobre el rol y la influencia del capital social sobre las condiciones de acceso a los servicios. En segundo lugar y tercer lugar se presenta la metodología y los resultados alcanzados. En la discusión de los resultados se aborda el análisis del capital social de los procesos de desarrollo de la APS como recomposición de la relación Estado sociedad y su capacidad para mejorar condiciones de acceso de los servicios, entendidos como problemática social que subyace en este tipo de políticas públicas.

\section{Capital Social y su aplicación a las políticas de APS.}

Antecedentes del capital social.

Como origen del desarrollo y utilización de capital social como categoría conceptual, se puede citar a Coleman ${ }^{1}$, quien aborda al capital social como parte de las características de la organización social, tales como confianza, normas y redes que facilitan la acción. Como alternativa, el capital social puede entenderse como permitiendo (o limitando) a los individuos a obtener acceso a diversos recursos, tales como ideas, información, dinero, servicios o favores, como resultado del funcionamiento de redes sociales, en donde el comportamiento de los actores y los modelos de participación son factores claves para producir tales productos ${ }^{2}$.

Este abordaje se restringe al supuesto de una relación causal positiva entre asociativismo de la comunidad y consecuencias sociales favorables y carecen de un análisis de la relación entre las lógicas y estructuras institucionales que definen el campo institucional, y las relaciones sociales que subyacen en el campo institucional.

Navarro V. ${ }^{11}$ señala que el concepto de capital social está centrado y limitado a una concepción de la comunidad como asociativismo de individuos. Según esta mirada crítica, la agenda tradicional de capital social ha desplazado el análisis necesario del juego de las relaciones de poder y cómo el poder se reproduce institucionalmente en términos ideológicos, culturales, políticos y económicos. De esta forma, se denuncia el riesgo de enfatizar el capital social como factor independiente de las políticas públicas, y por lo tanto la posibilidad de minimizar el debate del rol de los procesos sociales y políticos de las políticas y su capacidad para dar respuesta a las necesidades sociales. En otras palabras, Navarro ${ }^{11-12}$ denuncia las concepciones de capital social como condiciones necesarias para mejorar las condiciones sociales y la calidad de vida de la población, vacías del análisis del rol de las políticas púbicas como estrategias con capacidad para alterar la composición institucional de la relación entre Estado y sociedad. Según este argumento se sustenta en la tesis que sostiene que las formas de relación entre Estado y Sociedad determinan las formas de reproducción económica y social. Cualquier posibilidad de estudiar la solidaridad y la relación entre políticas y lo público, no puede ser entendida en un espacio de sociedad civil o capital social en forma aislada a las formas de articulación del Estado.

En la actualidad existe un mayor consenso hacia una concepción del capital social como 
categoría conceptual definida a partir de las relaciones y normas sociales estudiadas y su capacidad de influir sobre las condiciones sociales. En esta línea, Szreter y Woolcock ${ }^{13-14}$ explora dimensiones del capital social ("bonding" y bridging") para caracterizar la comunidad como formas instituidas de las "relaciones sociales". Con esta perspectiva, se aborda y pondera la noción de capital social a partir de las "relaciones" que expresan el tipo de vínculo entre los actores estratégicos que componen el campo institucional. Se incorpora de esta manera la noción de relaciones sociales entre actores con poder para influir en la composición de campos institucionales, y con influencia sobre las condiciones de acceso a los servicios sociales.

Woolcock ${ }^{15}$ y Szreter ${ }^{13-14}$ asignan un rol importante a la naturaleza de la relación entre estado y sociedad como un componente necesario de la teoría social que sustente el concepto de capital social. De esta forma, se destaca el rol relacional del capital social como una dimensión que permite caracterizar la naturaleza política e institucional de las relaciones entre la sociedad civil y el estado.

Según Szreter y Woolcock ${ }^{14}$ la característica relacional de capital social permite una discusión más integrada entre gobernanza y Estado. Según ellos, la teoría de capital social necesita introducir y articular el concepto de capital social con el análisis de las relaciones entre relaciones sociales y su relación con las políticas y estructuras del estado. Según esta perspectiva, el concepto de capital social relacional podría tener la utilidad de colaborar y complementar la descripción de las relaciones entre actores sociales y gubernamentales, reconociendo los aspectos de poder y construcción de nuevas normas sociales, con una problematización en función problemas sociales o sectoriales concretos. De tal forma, el capital social se constituye en una categoría relacional con capacidad de colaborar en el análisis de las relaciones sociales involucradas en las políticas públicas, con potencial explicativo de las políticas de extensión de la protección social en salud, que requieren de cambios en la relación entre sociedad civil y Estado. Para lograr mediar la relación entre nuevas relaciones sociales y cambios en capacidad de respuesta de políticas públicas, se requiere reconocer cuáles son los cambios institucionales que influyen sobre la efectividad de las mismas. En este marco, el concepto de campo institucional de los sectores sociales arroja luz sobre dimensiones que permitan reconocer los avances de las políticas sociales.

El sector salud y las políticas de APS.

En el caso del sector salud, el cambio de la relación entre Estado y sociedad es una dimensión medular para entender los cambios del campo institucional de los servicios de salud. Las limitaciones de los sistemas de salud en América Latina para garantizar condiciones de acceso de los servicios en función de las necesidades de salud de la población han sido explicadas por la fragmentación del modelo de organización, gestión y atención de los servicios públicos de salud.

La fragmentación del sistema de servicios ha sido caracterizada por la ausencia de mecanismos de coordinación entre diferentes organizaciones y servicios de salud, en donde el centro de la atención reside en hospitales, con presencia y poder protagónico de médicos especialistas, con 
mecanismos de regulación limitados a la autonomía profesional e individual de los profesionales. En estos casos, el Estado delega el poder de coordinación del sistema de servicios de salud a las corporaciones profesionales sin la presencia activa de otros actores sociales, que garanticen las condiciones de acceso a los servicios de salud.

Durante las últimas dos décadas, las políticas de APS en América Latina han procurado cambiar lógicas institucionales del campo institucional del sistema de servicios de salud, lo cual representa cambios relevantes en las relaciones sociales que integran la relación Estado y sociedad. Estos cambios han buscado producir cambios en la organización de los servicios de salud que favorecen el desarrollo de un primer nivel de atención, como medio para mejorar el acceso a los servicios de salud y como estrategia de coordinación del sistema de servicios de salud ${ }^{16}$.

Sin embargo, existen diferentes lógicas de APS, con diferentes alcances en el proceso de reconfiguración institucional del sistema de servicios de salud. En un extremo, perspectivas restrictivas de APS se han focalizado sobre una población específica, con prácticas de salud específicas, principalmente de atención de prevención, y promoción de la salud. Estas lógicas de APS se limitan al espacio y actores ubicados en el primer nivel de atención de la salud, sin modificaciones relevantes de las relaciones Estado y sociedad que conforman el campo institucional del sistema de servicios de salud. En contraste, lógicas de APS amplias y comprensivas, incluyen prácticas y espacios de intervención que atentan con las estructuras institucionales tradicionales, ya que alterna los modos de producción y la capacidad de respuesta del sistema de servicios a los problemas de acceso a los servicios de salud. La construcción de estas nuevas lógicas de APS disputan las lógicas institucionales del sistema de servicios de salud, con nuevas relaciones sociales establecidas entre aquellos actores que integran la relación del Estado y sociedad de este campo institucional ${ }^{17}$.

De esta forma, la producción de nuevas relaciones sociales en políticas de APS puede interpretarse en términos del desarrollo de capital social con capacidad de transformar el campo institucional del sistema de servicios de salud. En otras palabras, el capital social producido por políticas de APS, se interpreta considerando las nuevas relaciones sociales y los cambios institucionales con influencia sobre las condiciones de acceso a los servicios de salud.

\section{METODOLOGÍA}

El presente artículo tiene dos objetivos. En primer lugar, analizar cómo diferentes políticas de APS, configuran lógicas institucionales en las cuales subyacen diferentes tipos de capital social caracterizados por cambios en las relaciones sociales que son parte del entramado formas de relación entre el Estado y la sociedad civil. En segundo lugar, analizar la influencia del capital social subyacente en tales lógicas de APS en las condiciones de acceso a los servicios de salud.

Entre mayo de 2007 y septiembre de 2008 se condujo un estudio de casos múltiples con el 
propósito de construir una "teoría del proceso" que permita describir el desarrollo de las políticas de las APS en tres escenarios municipales: Rosario-Argentina, Vicente López-Argentina and Cochabamba-Bolivia. El abordaje metodológico está basado en tres estudios de casos, con un estudio retrospectivo que abarca el período de 1990 a 2008.

Los estudios de casos elegidos contienen diferentes políticas de APS, con características distintivas en las relaciones condiciones sociales y políticas establecidas entre los actores involucrados, la recomposición del campo institucional del sistema de servicios de salud y su influencia en las condiciones de acceso a los servicios de salud.

Se procura en este estudio abordar el "capital social" de las políticas de APS, como dimensión que configuran nuevas formas de relación entre Estado y sociedad civil, definida por nuevas relaciones sociales y espacios de interacción con influencia sobre la recomposición del campo institucional del sistema de servicios de salud. La recomposición del campo institucional del sistema de servicios de salud se caracteriza por las lógicas institucionales de APS. Con esta perspectiva, el estudio explora la relación entre "capital social" y condiciones de acceso a los servicios de salud.

Para ello fueron recolectados diferentes datos para abordar las diferentes dimensiones estudiadas. Las lógicas de APS y la construcción de su capital social subyacente fueron estudiadas a través de fuentes secundarias y primarias. Las fuentes secundarias utilizadas incluyen documentos oficiales -ej. organigramas y estadísticas-, publicaciones académicas y literatura gris. Los datos recolectados a través de fuentes primarias corresponden a entrevistas individuales a los directores de APS de Vicente López y Rosario, responsables de la gestión municipal y equipos técnicos de los tres casos (en total fueron realizadas 10 entrevistas en Rosario, 8 en Vicente López y 7 en Cochabamba), observaciones participantes y no participantes en reuniones e instancias de gestión, talleres con miembros de la dirección de APS y trabajadores de diversos CS y hospitales del municipio. También se realizaron observaciones en instancias de discusión del diseño y resultados de la investigación con autoridades y equipos de salud de la red de servicios de salud municipal. Las entrevistas y talleres fueron grabados y transcritos en su totalidad. Los textos fueron analizados utilizando la técnica de análisis de contenido.

Los cambios en las condiciones de acceso a los servicios fue realizado a través de un censo organizacional de centros de salud de primer nivel de atención (CS) municipales, lo cual permitió recolectar información sobre la apertura de centros de salud, la conformación de equipos de salud, su cobertura horaria, y la coordinación entre centros de salud y el resto del sistema de servicios de salud.

Este trabajo fue realizado gracias al apoyo del Centro Internacional de Investigaciones para el Desarrollo de Canadá (CIID). 


\section{RESULTADOS}

Los resultados de los casos presentan las dimensiones relevadas. En primer lugar, las características socio-demográficas y del sistema de servicios de salud de los municipios bajo estudio (Tabla 1). Posteriormente, se caracteriza el capital social de cada caso a partir de las relaciones sociales y la recomposición institucional de las iniciativas de APS (Tabla 2). Por último, se presentan las condiciones de acceso a los servicios de salud en los casos bajo estudio (Tabla 3).

Tabla 1: Características socio-demográficas y del sistema de servicios de salud de los municipios bajo estudio

\begin{tabular}{|l|l|l|c|}
\hline Municipio & Cochabamba & Vicente López & Rosario \\
\hline Habitantes* & 517.024 & 274.082 & 909.397 \\
\hline $\begin{array}{l}\text { Porcentaje de Población considerada pobre } \\
\text { estructural de acuerdo al índice de necesidades } \\
\text { básicas insatisfechas* }\end{array}$ & $33,8 \%$ & $4,8 \%$ & $13,5 \%$ \\
\hline Porcentaje de Población sin cobertura de salud* & $75,1 \%$ & $27,2 \%$ & $39,1 \%$ \\
\hline $\begin{array}{l}\text { Abordaje de APS } \\
\text { Tasa de Centros de Salud Públicos de primer nivel de } \\
\text { atención por cada 10.000 habitantes sin cobertura*** }\end{array}$ & 0,7 & Primer nivel & Integral \\
\hline $\begin{array}{l}\text { Médicos del 1er nivel de atención por cada 1000 } \\
\text { habitantes sin cobertura* ** }\end{array}$ & 0,3 & 2,6 & 1,4 \\
\hline $\begin{array}{l}\text { Hospitales públicos** } \\
\text { Fuente: }\end{array}$ & 5 & 1,2 & 14 \\
\hline $\begin{array}{l}\text { Argentina: Censo Nacional de Población, Hogares y Viviendas, 2001; Bolivia: Censo de Población y Vivienda, } \\
\text { 2001. } \\
* * \text { Secretarías de Salud municipales. }\end{array}$ & 6 & \\
\hline
\end{tabular}


Tabla 2: Capital Social: relaciones sociales y recomposición institucional de las iniciativas de APS de los casos bajo estudio.

\begin{tabular}{|c|c|c|c|c|c|}
\hline \multirow[b]{2}{*}{ Municipios } & \multicolumn{2}{|c|}{ Relaciones sociales (Estado - Sociedad) } & \multicolumn{3}{|c|}{ Recomposición institucional } \\
\hline & $\begin{array}{l}\text { Nuevos actores, } \\
\text { relaciones y } \\
\text { estructuras }\end{array}$ & $\begin{array}{l}\text { Relaciones de } \\
\text { Poder }\end{array}$ & $\begin{array}{l}\text { Alcance del } \\
\text { proceso de } \\
\text { cambio }\end{array}$ & Prestaciones & $\begin{array}{l}\text { Lógica de } \\
\text { APS }\end{array}$ \\
\hline Cochabamba & $\begin{array}{c}\text { Emergencia de } \\
\text { actores políticos } \\
\text { municipales y } \\
\text { movimientos sociales } \\
\text { con participación } \\
\text { formal en espacios } \\
\text { de gestión de nuevas } \\
\text { estructuras del } \\
\text { Estado (DILOS), } \\
\text { y resistencia de los } \\
\text { profesionales en } \\
\text { Hospitales. }\end{array}$ & $\begin{array}{l}\text { Liderazgo de } \\
\text { movimientos } \\
\text { sociales con } \\
\text { influencia sobre } \\
\text { equipos políticos } \\
\text { municipales y } \\
\text { especialistas } \\
\text { que conservan } \\
\text { su autonomía en } \\
\text { modelo de atención } \\
\text { y prácticas en los } \\
\text { Hospitales. }\end{array}$ & Periférica & $\begin{array}{l}\text { Paquete de } \\
\text { prestaciones } \\
\text { mínimas } \\
\text { con énfasis } \\
\text { en acciones } \\
\text { preventivas } \\
\text { dirigidas a } \\
\text { población } \\
\text { materno- } \\
\text { infantil. }\end{array}$ & $\begin{array}{l}\text { Transición de } \\
\text { un modelo } \\
\text { hospitalo- } \\
\text { céntrico hacia } \\
\text { una APS } \\
\text { Selectiva }\end{array}$ \\
\hline $\begin{array}{l}\text { Vicente } \\
\text { López }\end{array}$ & $\begin{array}{l}\text { Equipos de } \\
\text { ESAPS en alianza } \\
\text { con gestores y } \\
\text { equipo técnico de } \\
\text { la dirección de } \\
\text { APS, con relación } \\
\text { ambigua e inestable } \\
\text { con movimientos } \\
\text { sociales (gestión } \\
\text { compartida en CS) } \\
\text { y especialistas } \\
\text { de Hospitales } \\
\text { (coordinación en } \\
\text { espacios informales). }\end{array}$ & $\begin{array}{l}\text { Liderazgos de } \\
\text { ESAPS con } \\
\text { influencia limitada } \\
\text { al fortalecimiento } \\
\text { del primer nivel } \\
\text { de atención y } \\
\text { especialistas } \\
\text { conservando } \\
\text { autonomía del } \\
\text { modelo de atención } \\
\text { y prácticas en los } \\
\text { hospitales. }\end{array}$ & Intermedio & $\begin{array}{l}\text { Prestaciones } \\
\text { de prevención } \\
\text { y atención en } \\
\text { los centros de } \\
\text { salud. }\end{array}$ & $\begin{array}{c}\text { Transición } \\
\text { de una APS } \\
\text { Selectiva } \\
\text { hacia APS } \\
\text { como primer } \\
\text { nivel. }\end{array}$ \\
\hline Rosario & $\begin{array}{c}\text { Participación y } \\
\text { colaboración entre } \\
\text { Equipos de ESAPS, } \\
\text { especialistas, } \\
\text { actores políticos y } \\
\text { técnicos municipales } \\
\text { y movimientos } \\
\text { sociales en } \\
\text { diferentes espacios } \\
\text { de participación } \\
\text { en distritos } \\
\text { descentralizados } \\
\text { (Distritos de APS y } \\
\text { distritos de Salud) }\end{array}$ & $\begin{array}{c}\text { Liderazgo de } \\
\text { ESAPS con } \\
\text { influencia sobre } \\
\text { espacios de } \\
\text { conducción, gestión } \\
\text { y coordinación } \\
\text { del sistema de } \\
\text { servicios de salud } \\
\text { y el modelo de } \\
\text { atención. }\end{array}$ & Central & $\begin{array}{l}\text { Gestión de la } \\
\text { continuidad } \\
\text { de la atención } \\
\text { integral entre } \\
\text { diferentes } \\
\text { niveles de } \\
\text { atención }\end{array}$ & $\begin{array}{l}\text { Transición } \\
\text { hacia una } \\
\text { estrategia de } \\
\text { APS como } \\
\text { estrategia de } \\
\text { coordinación } \\
\text { del sistema de } \\
\text { servicios de } \\
\text { salud. }\end{array}$ \\
\hline
\end{tabular}

Fuente: elaboración propia en base fuentes primarias (entrevistas y talleres) y secundarias (revisión documental) producidas en el marco de la investigación.

\section{Referencias:}

CS: Centros de Salud

DILOS: Directorios Locales de Salud.

ESAPS: Equipos de Salud de Atención Primaria de la Salud. 
Tabla 3. Condiciones de acceso a los servicios de salud en los casos bajo estudio

\begin{tabular}{|c|c|c|c|c|c|c|c|}
\hline \multirow[b]{2}{*}{ Municipio } & \multicolumn{2}{|c|}{$\begin{array}{c}\text { Cantidad de centros de } \\
\text { salud* }\end{array}$} & \multicolumn{2}{|c|}{$\begin{array}{l}\% \text { de CS con equipos } \\
\text { multi-disciplinarios. } \\
* *++\end{array}$} & \multicolumn{2}{|c|}{$\begin{array}{l}\text { Horario de atención por } \\
\text { semana (promedio)** } \\
\text { *** }\end{array}$} & \multirow{2}{*}{$\begin{array}{l}\text { Coordinación. } \\
\% \text { de CS que } \\
\text { gestionan las } \\
\text { referencias de } \\
\text { sus pacientes } \\
\text { a hospitales } \\
\text { y servicios } \\
\text { especializados }\end{array}$} \\
\hline & $\begin{array}{l}\text { Al inicio del } \\
\text { proceso de } \\
\text { reforma**** }\end{array}$ & 2008 & $\begin{array}{l}\text { Al inicio del } \\
\text { proceso de } \\
\text { reforma**** }\end{array}$ & 2008 & $\begin{array}{c}\text { Al inicio del } \\
\text { proceso de } \\
\text { reforma**** }\end{array}$ & 2008 & \\
\hline Cochabamba & 6 & 28 & $0 \%$ & $0 \%$ & $\begin{array}{l}\text { Reducido e } \\
\text { inestable }\end{array}$ & 35,3 & $0 \%$ \\
\hline Vicente López & 7 & 20 & $0 \%$ & $60 \%$ & $\begin{array}{l}\text { Reducido e } \\
\text { inestable }\end{array}$ & 46,9 & $30 \%$ \\
\hline Rosario & 49 & 78 & $0 \%$ & $73,8 \%$ & $\begin{array}{l}\text { Reducido e } \\
\text { inestable }\end{array}$ & 59,3 & $100 \%$ \\
\hline
\end{tabular}

Fuente: elaboración propia en base a:

* Registros de las secretarías de salud municipales.

** Censo Organizacional: Rosario, 2007; Vicente López y Cochabamba, 2008.

$* * *$ Entrevistas

**** Año de inicio de cada reforma: Cochabamba 1993; Vicente López 1993; Rosario 1990.

++ Se consideró que la composición era multidisciplinaria cuando en el equipo existiera al menos:

- En Argentina: un generalista o pediatra, un clínico, ginecólogo, toco ginecólogo u obstetra; un enfermero y un trabajador social o un psicólogo.

- En Bolivia: un médico general, un odontólogo, una enfermera licenciada, y una enfermera auxiliar de campo.

CS: Centros de Salud. 
Cochabamba.

\section{Capital Social}

Los cambios en la relación Sociedad - Estado se basan en una mayor participación de los movimientos sociales en espacios de gestión del Estado, y la emergencia de equipos técnicos y políticos locales a nivel municipal, pero con resistencia y rechazo de las organizaciones profesionales, que conservan su poder de influencia sobre la organización del sistema de servicios de salud.

\section{Campo Institucional}

Los cambios en las relaciones sociales tienen un efecto marginal en el campo institucional del sistema de servicios de salud. La lógica de APS promovida es restringida a prestaciones de prevención y promoción de la salud, con cambios marginales en las condiciones de acceso en el primer nivel de atención y con médicos especialistas que conservan su poder de influencia en el modelo de atención en los hospitales y en el sistema de servicios de salud.

\section{Condiciones de Acceso}

En cuanto a cambios en las condiciones de acceso a los servicios de salud, si bien se abren 28 nuevos centros de salud, no se producen cambios sustantivos en tales servicios, ni en la cobertura horaria, ni en la conformación de equipos de salud.

\section{Vicente López}

\section{Capital Social}

Los cambios en la relación Sociedad Estado se construyen en torno a una mayor participación de un grupo de profesionales conformado por médicos generalistas y enfermeras comprometidas con el desarrollo de APS, equipos técnicos de la dirección de atención primaria de la salud, y coordinadores de los programas de salud y los profesionales de los centros de salud. La relación con los movimientos sociales es inestable, ya que éstos gestionan sus propios centros de salud con dificultades en la aceptación de normas técnicas de gestión y atención. La relación con especialistas de los hospitales es conflictiva, que éstos conservan poder e influencia de su práctica, con mecanismos de coordinación sólo informales. La relación con los políticos locales es híbrida, ya que si bien asumen una posición favorable general, no se comprometen cuando entran en colisión con intereses y concepciones de los especialistas ubicados en los hospitales, o a las presiones de los movimientos sociales.

\section{Campo institucional}

El cambio en el campo institucional alcanza un nivel intermedio, limitado al desarrollo de una lógica de APS centrada en el desarrollo del primer nivel de atención en los centros de salud. 
Inicialmente se parte con la incorporación de prácticas de prevención, con programas materno infantiles y extendidos, y llegan a un amplio abanico de programas de salud, con la incorporación de servicios de recuperación de la salud, ofreciendo una cobertura más integral de servicios en los centros de salud. Sin embargo, los hospitales y especialistas conservan su poder relativo y autonomía en su modelo de atención y organización de servicios.

\section{Condiciones de Acceso}

Los cambios la organización de los centros de salud está centrado en un aumento de 20 centros de salud, con una proporción de los mismos gestionada por los movimientos sociales. Sin embargo, la conformación de equipos de salud es heterogéneo y limitaciones en la cobertura horaria y en adscripción de la población a los servicios de salud.

\section{Rosario}

\section{Capital Social}

Los cambios en la relación sociedad civil y Estado se producen utilizando como plataforma nuevas estructuras del Estado municipal (colegiados de gestión, distritos de APS y distritos de salud) que habilitan la interacción del grupo de profesionales comprometidos con la APS con movimientos sociales, políticos locales, y gestores y profesionales de hospitales.

\section{Campo Institucional}

La recomposición del campo institucional del sistema de servicios de salud tiene un alcance central. Si bien en una primera etapa, se producen cambios limitados a la ampliación de prácticas de prevención y promoción de salud en los centros de salud, posteriormente asume un rol de garantía de las condiciones de acceso a todas las prestaciones del sistema de servicios de servicios de salud y de coordinación del proceso de atención entre los diferentes niveles de atención. Estos cambios han introducido nuevas formas de regulación y organizacional del sistema de servicios de salud.

\section{Condiciones de Acceso}

Los cambios en el sistema de servicios de salud se expresan en un aumento de los centros de salud, con presencia mayoritaria de equipos interdisciplinarios, una ampliación de cobertura horaria y en condiciones de coordinación con el segundo nivel de atención.

\section{CONCLUSIONES}

La producción de "capital social" en las políticas de APS es interpretada como innovaciones en las relaciones sociales producidas en los procesos de reconfiguración de la relación Estado Sociedad en los espacios locales. Estos cambios tienen influencia sobre la efectividad de las 
políticas de salud en la medida que logran transformaciones del campo institucional del sistema de servicios de salud ${ }^{16,18}$.

De esta forma, el capital social, deja de ser concebido como un atributo institucional exógeno a los procesos sociales y políticos, y por el contrario se asume como dimensión clave y parte del proceso políticos orientadas al desarrollo de sistemas de protección social ${ }^{19}$. El análisis de las diferentes concepciones de $\mathrm{APS}^{17}$ ofrece una oportunidad para desentrañar cómo diferentes lógicas institucionales se vinculan con relaciones sociales que explican la capacidad de las políticas públicas para abordar los problemas sociales. En otras palabras, el capital social contribuye a explicar la relevancia e influencia de los procesos sociales mismos como parte y soporte de los cambios institucionales que modelan la efectividad de las políticas de salud.

El análisis de los casos permite ponderar algunos hallazgos en términos de los procesos de cambio de la relación Estado sociedad que subyacen en la implementación de diferentes lógicas de APS y sus efectos en términos de acceso a los servicios.

En los tres casos existen políticas locales de nuevas lógicas institucionales de APS que se enmarcan y sustentan por la emergencia de nuevas formas de relación entre Estado y Sociedad. Los cambios de nuevas formas de relación de Estado y Sociedad se expresa por la incorporación de nuevos actores de la sociedad civil y del Estado, y la producción de nuevas estructuras del Estado que representan espacios en los cuales se produce la participación y las nuevas relaciones entre los actores involucrados. Sin embargo, cada uno de los casos tiene diferencias significativas, en la influencia de las nuevas formas de relación entre Estado y sociedad civil está mediatizado por el tipo de influencia sobre la recomposición institucional del sistema de servicios de salud.

En Cochabamba, la emergencia de un capital social que conjuga cambios significativos con nuevas relaciones sociales entre movimientos sociales y actores políticos locales se realizan en espacios formales de participación del Estado municipal, con incidencia en procesos de decisión en la asignación de recursos y gestión de servicios de salud.

Sin embargo, aunque estos cambios son relevantes, los procesos de reconfiguración institucional con los cuales se relacionan explican su bajo nivel de efectividad, ya que estas iniciativas promueven lógicas de APS restringidas a servicios de prevención, sin incidencia sobre la organización y modelo de atención de los hospitales, en donde los especialistas sostienen una posición de resistencia a los cambios, conservando autonomía y autorregulación de sus prácticas de salud.

En Vicente López, la configuración del capital social está determinado por la conformación de equipos de profesionales comprometidos e involucrados en las iniciativas de APS, ubicados en diferentes nuevos espacios del Estado creados durante este proceso: la dirección de APS, los centros de salud y la coordinación de programas de prevención.

Las relaciones inestables y frágiles en términos de colaboración con movimientos sociales, 
políticos locales y especialistas, y la informalidad de los mecanismos de coordinación con servicios del hospital debilitan la influencia de esta iniciativa más allá de mejoras limitadas en el primer nivel de atención.

En Rosario, el proceso de implementación de lógicas de APS se sustenta por el desarrollo de un capital social que adquiere diferentes tipos de relaciones sociales y nuevos espacios formales de interacción. Las relaciones sociales son colaboración y alianzas entre el equipo de profesionales comprometidos con esta iniciativa, con diferentes actores (movimientos sociales, actores políticos locales, equipos técnicos y médicos especialistas). Los espacios de interacción son nuevas estructuras del Estado municipal toman la forma de colegiados de gestión en CS, distritos de APS y distritos de salud. En todos los casos, tales espacios albergan la participación e interacción de los actores involucrados.

Los mayores alcances de las iniciativas de APS en Rosario se explican por dos características distintivas del capital social de este caso. En primer lugar, existen espacios de interacción, tales como colegiados de gestión y distritos de APS en donde se fortalece la colaboración entre profesionales que integran equipos de APS con movimientos sociales, lo cual favorece la legitimidad social y política de estos procesos de cambio. En segundo lugar, profesionales de primer nivel de atención logran acuerdos con especialistas y gestores de hospitales a través de los distritos de salud, facilitando la legitimidad profesional y la viabilidad de la influencia de lógicas de APS sobre la organización de todos los niveles del sistema de servicios de salud.

Estos hallazgos permiten reconocer la influencia de la configuración entre Estado y sociedad para modelar las relaciones de poder que subyacen al proceso de implementación de las reformas del sector salud y sus resultados ${ }^{19-20}$.

\section{BIBLIOGRAFÍA}

1. Coleman JS. Social capital in the creation of human capital. Am J Social. 1988;94:S95-121.

2. Portes A. Social capital: its origins a aplications in moderm sociology. Annu Rev Sociol. 1998;24:1-24.

3. Putnam RD. Making democracy work: civic traditions in modern Italy. Princeton: Princeton University Press; 1993. 229 p.

4. Portes A. social capital: its origins and applications in modern sociology. Annu Rev social. $1998 ; 24: 1-24$.

5. Whitehead M, Diderichsen F. Social capital and health: tip-toeing through the minefield of 
evidence. Lancet. 2001;358:165-66.

6. Lynch JW, Due P, Muntaner C, Smith GD. Social capital: is it a good investment strategy for public health? J Epidemiol Community Health. 2000 Jun; 54(6): 404-8.

7. Siegrist J. Social capital and health. Gesundheitswesen. 2002;64:189-92.

8. Lomas J. Social capital and health: implications for public health and epidemiology. Soc Sci Med. 1998;47:1181-88.

9. Morrow V. Conceptualising social capital in relation to the well-being of children and young people: a critical review. Sociol Rev. 1999;47:744-65.

10. Hawe P, Shiell A. Social capital and health promotion a review. Soc Sci Med. 2000;51:871-85.

11. Navarro V. A critique of social capital. Int J Health Serv. 2002;32:423-32.

12. Navarro V, Shi L. The political context of social inequalities and health. Int J Health Serv. 2001;31:1-21.

13. Szreter S. The state of social capital: bringing power, politics and history back. Theory and Society. 2002;31:573-621.

14. Szreter S, Woolcock M. Health by association? Social theory, and the political economy of public health. Int J Epidemiol. 2004;33:650-67.

15. Woolcock M. The place of social capital in understanding social and economic outcomes. Can J Policy Res. 2001;2:11-17.

16. Báscolo, Ernesto. Gobernanza y economía política de las políticas de APS en América Latina. Cien Saude Colet, 2011, vol. 16, no 6, p. 2763-2772.

17. Báscolo, Ernesto. Gobernanza de las organizaciones de salud basados en Atención Primaria de Salud. Revista de salud pública, 2010, vol. 12, p. 8-27.

18. Manciko J, Stardfield B. The utility of social capital in research on health determinants. Milbank Q. 2001;79:387-427.

19. Navarro V. Commentary: is capital the solution or the problem? Int J Epidemiol. 2004; 
$33: 672-4$

20. Cattel V. Poor people, poor places, and poor health: the mediating role of social networks and social capital. Soc Sci Med. 2001;52:1501-16.

Artigo apresentado em 30-09-15

Artigo aprovado em 25-02-16

Artigo publicado no sistema em 30-03-16 